\title{
Deterministic dynamics of the magnetosphere: results of the 0-1 test
}

\author{
S. Prabin Devi ${ }^{1}$, S. B. Singh ${ }^{1}$, and A. Surjalal Sharma ${ }^{2,3}$ \\ ${ }^{1}$ Department of Physics, Manipur University, Canchipur, Imphal - 795003, India \\ ${ }^{2}$ Department of Astronomy, University of Maryland, College Park, MD 20742-2421, USA \\ ${ }^{3}$ Research Institute of Science and Technology, D.M. College of Science Complex, Imphal - 795001, India \\ Correspondence to: S. B. Singh (brajamanisanasam@gmail.com)
}

Received: 1 June 2012 - Revised: 4 December 2012 - Accepted: 10 December 2012 - Published: 7 January 2013

\begin{abstract}
A test for deterministic dynamics in a time series data, namely the $0-1$ test (Gottawald and Melbourne, 2004, 2005), is used to study the magnetospheric dynamics. The data, corresponding to the same time period, of the auroral electrojet index $A L$ and the magnetic field component $B_{z}$ of the solar wind magnetic field measured at $1 \mathrm{AU}$ are used to compute the parameter $K$, which is zero for non-chaotic and unity for chaotic systems. For the magnetosphere and also for the turbulent solar wind, $K$ has values corresponding to a nonlinear dynamical system with chaotic behaviour. This result is consistent with the Lyapunov exponents computed from the same time series data.
\end{abstract}

\section{Introduction}

The coupled solar wind-magnetosphere system is a highly variable dynamical system with continuous exchanges of mass, momentum and energy through many plasma processes, including magnetic reconnection (Vasyliunas, 1975). As a consequence of the continuous solar wind driving, the magnetospheric dynamics is far from equilibrium and has been described as a nonlinear system that exhibits complex and irregular behaviour (Sharma, 1995; Klimas et al., 1996). The complexity of the magnetosphere has been extensively studied using different approaches. The first studies were based on the autonomous behaviour of the magnetosphere and thus used the data of the magnetosphere alone (Vassiliadis et al., 1990, 1991; Shan et al., 1991; Price and Prichard, 1993; Sharma et al., 1993; Sharma, 1995), and emphasized its low-dimensional behaviour. Recognizing the driven nature of the magnetosphere, the next stage of studies considered the solar wind and magnetosphere as an inputoutput system (Price et al., 1994; Vassiliadis et al., 1995;
Valdivia et al., 1996; Chen and Sharma, 2006). Such models capture the dynamics of magnetosphere, especially during magnetic storms and substorms. The magnetospheric substorm is a process in which energy stored in the magnetotail through solar wind-magnetosphere interaction is released explosively, causing dramatic phenomena in various regions of the magnetosphere and ionosphere. This coupling is strongly enhanced when the interplanetary magnetic field turns southward (Akasofu, 1981). A typical substorm, with a time scale of an hour, consists of three phases, viz.: energy storage in the magnetotail (growth phase), sudden unloading or release of the stored energy (expansion phase), and a return toward the quasi-equilibrium state (recovery phase). Among the geomagnetic indices representing magnetospheric phenomena, the auroral electrojet indices $A U$, $A L$, and $A E$ (Mayaud, 1980), derived from the magnetic field variations in the high-latitude auroral zone, closely reflect the features of magnetospheric substorms. During periods of enhanced geomagnetic activity the westward electrojet, monitored by the $A L$ index, increases abruptly due to currents driven by plasma processes in the magnetotail. On the other hand, the eastward electrojet, which is monitored by the $A U$ index, increases due to processes such as the partial ring current closure via the ionosphere in the evening sector (Feldstein et al., 2006). Therefore, the analysis of the $A L$ and $A U$ indices can yield insights into the dynamics of different aspects of the magnetosphere. The $A L$ index reflects the variability of the magnetospheric substorms and is widely used in the studies of the dynamical behaviour, as in this paper. On the longer time scales, typically $10 \mathrm{~h}$, the magnetic storms are the dominant phenomenon and the storm-time substorms are most intense (Sharma et al., 2003).

The theory of nonlinear, deterministic dynamical systems provides a powerful approach to the study of large-scale 
natural systems, such as the Earth's magnetosphere. The techniques of phase-space reconstruction (Abarbanel et al., 1993; Kantz and Schrieber, 1997) yield the dynamical features, such as low dimensionality and characteristic time scales, inherent in the observational time series data. A key feature of this approach is its ability to yield these dominant dynamical properties inherent in the data, independent of modelling assumptions. This has stimulated many applications to the time series data of natural and anthropogenic systems, and has provided an improved understanding of the underlying dynamics. During the last two decades, the nonlinear time series methods have been used to study the magnetosphere using $A E$ and $A L$ index time series, and these studies have shown evidence of chaotic behaviour in the magnetospheric dynamics. The first study (Vassiliadis et al., 1990) used the auroral electrojet index $A E$ for the reconstruction of phase space and found good evidence in support of the low dimensionality of the magnetospheric dynamics. This was followed by a large number of studies using improved phasespace reconstruction techniques such as the singular spectrum analysis (Sharma et al., 1993; Pavlos et al., 1994). The scaling properties of the magnetosphere have also been studied, for example, in the form of multifractal analysis, self organized criticality and intermittency (Consolini et al., 1996; Chang, 1999; Klimas et al., 2000; Freeman et al., 2000; Sitnov et al., 2000, 2001). The low dimensionality of the magnetospheric dynamics is mainly a geometrical property and enables the modelling of the dynamics using a small number of variables. On the other hand the Lyapunov exponents are directly related to the divergence of the neighbouring trajectories and thus to the chaotic behaviour. The computations of these exponents from the time series data of the magnetosphere (Vassiliadis et al., 1991; Pavlos et al., 1999) yield at least one positive Lyapunov exponent, thus showing the presence of chaos.

The low dimensionality and chaotic behaviour derived from reconstruction of phase space from time series data using time delay embedding (Abarbanel et al., 1993; Kantz and Schrieber, 1997) are subject to uncertainties, due mainly to the limitations on the data and multiscale phenomena (Ukhorskiy et al., 2004), in yielding clear dynamical trajectories. In the studies using the auroral electrojet indices, the phase space reconstructed from the data and its surrogates were found to be similar (Price and Prichard, 1993; Price et al., 1994). Similarly, the computation of the Lyapunov exponent is subject to uncertainties. Although the improvements in the phase-space reconstruction, such as orthonormality obtained by using singular vectors (Sharma et al., 1993), have led to better results, it is important to use independent techniques for a better understanding of the magnetospheric dynamics. In this paper, the $0-1$ test for chaos (Gottawald and Melbourne, 2004, 2005) is used to study the deterministic, chaotic behaviour of magnetospheric substorms using correlated data of the solar wind-magnetosphere system. For the magnetosphere the $A L$ index data for the years 2001 and
2007, which correspond to the peak periods of the last solar maximum and minimum, respectively, are used. This choice of the periods of extrema in solar activity provides, in addition to the study of the deterministic features, a means to study the differences in general. Since the storms and substorms are responses of the magnetosphere to the solar wind driver, the north-south $z$ component of the interplanetary magnetic field (IMF), responsible for magnetic reconnection at the magnetopause, is used as a variable representing the solar wind. The analysis based on the $0-1$ test is thus carried out on the IMF $B_{z}$, measured at $1 A U$, and $A L$, during the same periods. So far the $0-1$ test for chaos has been applied to simulated data such as those generated from the integration of Lorenz equations and from laboratory experiments (Falconer et al., 2007; Chowdhury et al., 2012). This paper presents the first application of the $0-1$ test to the observational data of a natural system. In order to validate the results obtained by this test, the Lyapunov exponents are computed using the standard techniques (Hegger et al., 1999) and the consistency among the results analyzed.

The paper is organized as follows: The next section is a brief description of the $0-1$ test algorithm used in the analysis of the magnetospheric data. In Sect. 3 the correlated solar wind and magnetospheric data and the analysis of the dynamical behaviour using the $0-1$ test, as well as comparison with results obtained from the computation of Lyapunov exponents, are presented. The main results and their implications are summarized in the concluding Sect. 4.

\section{Test for deterministic dynamics in time series data}

The ubiquity of complex or irregular behaviour in time series data of many natural and anthropogenic systems has stimulated the development of techniques to characterize the inherent dynamical features. The most widely used techniques are the different forms of phase-space reconstruction using the time delay embedding (Abarbanel et al., 1993). Implicit in this approach are the reconstructed trajectories, and many studies have been developed to improve the reconstruction (Kaplan and Glass, 1992; Kennel et al., 1992; Wayland et al., 1993). However, in many cases the dynamical features, such as trajectories, are not obtained clearly and this limits the ability of the techniques to yield conclusive results.

The $0-1$ test (Gottawald and Melbourne, 2004, 2005) for characterizing the origins of the irregularity of a time series overcomes this limitation and thus provides an effective and independent technique. This test is based on two main components. In the first given time series data are used to drive the dynamics on a well-chosen group extension. The second is a theorem (Nicol et al., 2001) that states that the dynamics on the group extension is bounded if the underlying dynamics is non-chaotic, but is unbounded and sub-linear if the dynamics is chaotic. The time series is used to compute a parameter $K$, which can have values in the range $0 \leq K \leq 1$, 
with the value of zero for non-chaotic and unity for chaotic systems.

This method has many advantages over the methods of computing the largest Lyapunov exponents as signatures of chaos underlying complex time series data, arising mainly from the property that it circumvents the need for phasespace reconstruction. Moreover the form, nature and dimension of the underlying dynamics are irrelevant for the test; i.e. they do not pose practical limitations on the method as is the case for traditional methods involving phase-space reconstruction. This method applies equally well to continuous time or discrete time systems, to experimental or computed data, and to data from ordinary or partial differential equations. The test is robust, in specific cases, to contamination by noise; that is, it can cope with the presence of significant amount of noise in the data. All that is required are time series data of an observation of the dynamics sampled at regular intervals; almost any reasonable function of the state of the system can be used. This makes the 0-1 test an especially easy test to implement. The test determines if a variable (to be defined later as $p\left(t_{\alpha}\right)$ ), derived from the time series data, corresponds to Brownian motion. This test has been applied successfully to many known deterministic systems and showed near-perfect correlation between this test and the standard methods of computing Lyapunov exponents. For example, this test has been applied to the well-known Henon-Heiles and Lorenz systems, and found to be useful as a marker of the transition from regularity to chaos (Barrow and Levin, 2003). The application to the time series data from laboratory experiments has shown its utility as an analysis tool (Falconer et al., 2007; Chowdhury et al., 2012). These results support the claim (Gottawald and Melbourne, 2004) that the dimension of the dynamical system and the nature of the underlying equations are not directly relevant, since the $0-1$ test does not require the phase-space reconstruction of conventional nonlinear time series methods (Abarbanel et al., 1993; Hegger et al., 1999). Sun et al. (2010) illustrated the selection of parameters of this algorithm by numerical experiments and also validated the reliability and the universality of the test algorithm, by applying to typical nonlinear dynamical systems, including fractional-order dynamic system.

The $0-1$ test can be implemented readily for a given datum $\phi\left(t_{\alpha}\right)$ at time $t_{\alpha}(\alpha=1,2,3, \cdots N)$ with a fixed interval and representing the underlying dynamics. Choosing a random constant $c \in \mathbf{R}$, a real number ( 0.7 in this study), a function $p\left(t_{\alpha}\right)$, is defined as

$p\left(t_{\alpha}\right)=\sum_{j=1}^{\alpha} \phi\left(t_{j}\right) \cos (j c)$.

To characterize the growth of the function defined in Eq. (1), the test uses the mean square displacements. For Brownian motion, the average of $\left[p\left(t_{j+\alpha}\right)-p\left(t_{\alpha}\right)\right]$ is expected to grow as $\sqrt{t_{\alpha}}$ for $\alpha=1,2,3, \ldots, N$. Therefore, the square of $\left[p\left(t_{j+\alpha}\right)-p\left(t_{\alpha}\right)\right]$ should asymptotically approach linear growth with $t_{\alpha}$ for sufficiently large $N$. For a finite length of data, the mean square displacement is defined as

$M\left(t_{\alpha}\right)=\lim _{N \rightarrow \infty} \frac{1}{N-\alpha} \sum_{j=1}^{N-\alpha}\left[p\left(t_{j+\alpha}\right)-p\left(t_{\alpha}\right)\right]^{2}$.

This definition of mean square displacement requires $1 \ll \alpha \ll N$, which implies that $M\left(t_{\alpha}\right)$ grows linearly in time if $\alpha$ is in the range $1 \ll \alpha \ll N$; practically best results are obtained when $\alpha \leqslant N / 10$. This test is based on the recognition that $M\left(t_{\alpha}\right)$ is bounded in time for regular dynamics while scaling linearly with time for chaotic dynamical features. Thus the scaling behaviour of $M\left(t_{\alpha}\right)$ is the essential element for the test, and is computed from its asymptotic growth rate $K$ defined as

$K=\lim _{\alpha \rightarrow \infty} \frac{\log \left(M\left(t_{\alpha}\right)\right)}{\log \left(t_{\alpha}\right)}$.

In the computations $\log \left(M\left(t_{\alpha}\right)+1\right)$ is used to avoid negative logarithms. For a time series of finite length, $K$ is computed by performing a least square fit of $\log \left(M\left(t_{\alpha}+1\right)\right)$ versus $\log \left(t_{\alpha}\right)$, in the range $1 \leqslant \alpha \leqslant N / 10$. The processes and the underlying complexity are then characterized as nonchaotic for $K \simeq 0$ and chaotic if $K \simeq 1$. In practice the computed values of $K$ for most time series data are expected to lie between these two limits.

The application of this test to well-known systems has demonstrated the validity of this technique. For the eightdimensional Lorenz system (Lorenz, 1996), $K$ has values in the range 0.7-0.8 (Gottawald and Melbourne, 2005). Considering that the Lorenz system is clearly chaotic, this indicates that the cases of actual systems, which are chaotic the $K$ values, are likely to be smaller due to the presence of noise and non-chaotic features.

\section{Deterministic dynamics of the coupled solar wind-magnetosphere system}

The magnetospheric conditions vary widely depending on the intensity of the solar wind driver, and its dynamical characteristics may reflect this dependency, at least partly. In general, the two limits in the range of activities are expected to correspond to the solar maximum and minimum. Keeping this in mind the years 2001 and 2007, which correspond to the last solar maximum and minimum, respectively, are chosen for this study. Among the various databases of the plasma and field variables of the magnetosphere, the auroral electrojet indices have been used extensively in the study of its dynamical behaviour. The index $A L$ reflects the magnetospheric variability; considering substorm time scales of $\sim 1 \mathrm{~h}$, the 5min averaged data are used.

The solar wind data provided by ACE spacecraft, at the first Lagrange point $(L 1)$ upstream of the magnetopause, provide time series of the plasma and field variables driving the 


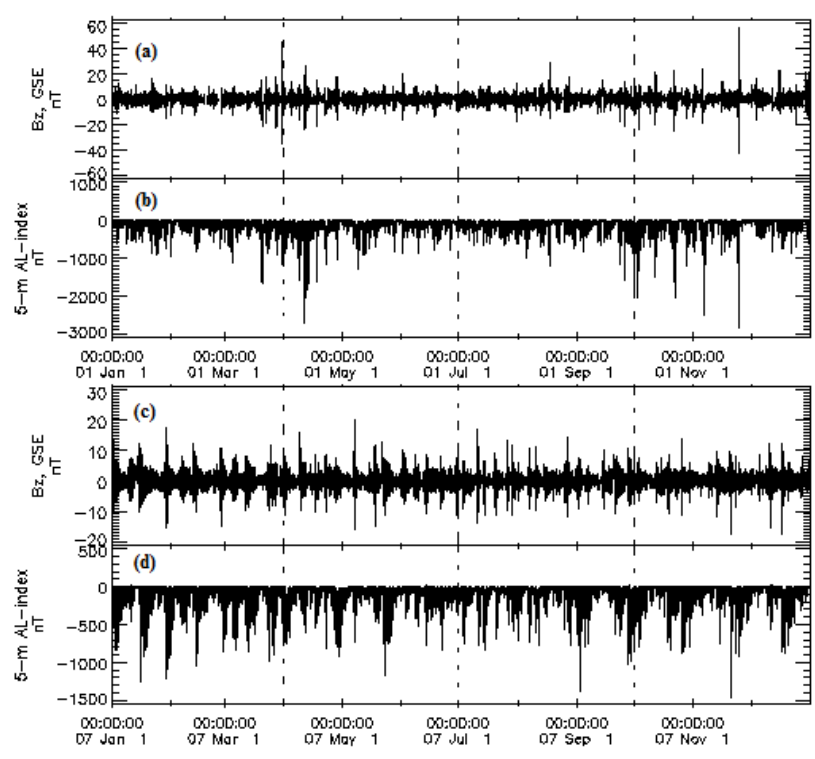

Fig. 1. The component $B_{z}$ of IMF and auroral electrojet index $A L$ for the years 2001 (a and b) and 2007 (c and d). The dashed vertical lines mark the three-month periods or quarters.

magnetosphere. The coupling of the solar wind to the magnetosphere is enhanced during periods when the interplanetary magnetic field (IMF) is mainly southward, and thus the $z$-component $B_{z}$ is considered the primary driver of magnetospheric activity. The 5-min averaged IMF $B_{z}$ data at $1 A U$ (ACE data) during the same periods as the $A L$ time series are used for the analysis. The aim is to identify if the nonlinear chaotic behaviour of the magnetosphere is indeed inherent in its dynamics or is a direct response to the solar wind driver. The $A L$ and $B_{z}$ used in this study have been obtained from the OMNI database (Combined $1 A U$ IP Data; Magnetic and Solar Indices; http://spdf.gsfc.nasa.gov).

The IMF $B_{z}$ and $A L$ index are shown in Fig. 1 for the two years 2001 (panels a and b) and 2007 (panels c and d). For each year the data are divided into four quarters (marked by the dashed vertical lines). The differences in the activity levels in these two cases are evident in both the IMF and magnetospheric data (note the different scales for the two years). The $A L$ variations for the intense geomagnetic substorms have very high values, reaching close to $3000 \mathrm{nT}$ in a few cases during 2001. During 2007, the quiet period near the last solar minimum, the peak $A L$ values are reduced by a factor close to two. It should however be noted that the analysis presented in this paper focuses on the overall behaviour of the dynamical system in these two periods.

For each of the data segments, the values of $K$ are computed using Eqs. (1)-(3). Although the length of each time series data should be very large, there are practical considerations that limit the data size used in the computations. As the algorithm involves computational loops, larger data sequences lead to longer loops and the execution time increases
Table 1. The $K$ values of the $0-1$ test for chaos and the corresponding Lyapunov exponents, computed using the Hegger et al. (1999) algorithm, for the 3-month epochs of 2001 and 2007.

\begin{tabular}{ccccccc}
\hline Year & Epoch & $\begin{array}{c}\text { Time series } \\
\text { length } N\end{array}$ & $\begin{array}{c}K \\
(A L)\end{array}$ & $\begin{array}{c}K \\
\left(B_{z}\right)\end{array}$ & $\begin{array}{c}\text { LE } \\
(A L)\end{array}$ & $\begin{array}{c}\text { LE } \\
\left(B_{z}\right)\end{array}$ \\
\hline \multirow{2}{2}{001} & Jan-Mar & & 0.7596 & 1.0949 & 0.1313 & 0.1110 \\
& Apr-Jun & & 0.8320 & 0.8101 & 0.1255 & 0.1052 \\
& Jul-Sep & & 0.8159 & 0.7940 & 0.1219 & 0.0983 \\
& Oct-Dec & 20000 & 0.7558 & 0.9844 & 0.1227 & 0.0855 \\
\hline 2007 & Jan-Mar & & 0.8335 & 0.9171 & 0.1016 & 0.0914 \\
& Apr-Jun & & 0.9352 & 0.9760 & 0.1080 & 0.1027 \\
& Jul-Sep & & 0.8153 & 1.0071 & 0.1063 & 0.0986 \\
& Oct-Dec & & 0.7752 & 0.6946 & 0.0964 & 0.0905 \\
\hline
\end{tabular}

significantly, thereby decreasing the efficiency of the implementation significantly. Taking all of the above into consideration, an approximate value of the sequence length $N$ should be selected so that the data explore enough regions of the attractor. The greater the $N$ is, the closer to ideal value of $K$. The plots of $K$ as a function of the time series length $N$ are shown in Fig. 2. It is seen that in all the cases the values of $K$ initially show a linear increase with $N$ and converge to finite values for large $N$. Many runs of the algorithm were carried out for data sequences of variable lengths, and it was observed that values of $K$ are reasonably constant for $N \geq 15000$. This clearly indicates that proper coverage of the state space occurs for $N \geq 15000$. Figure 3 shows the plot $\log M\left(t_{\alpha}\right)$ vs. $\log \left(t_{\alpha}\right)$ for the third quarters of 2001 and 2007 for the $A L$ indices and IMF $B_{z}$ calculated with 20000 points, and the corresponding $K$ values are shown. These values, close to unity, clearly indicate the presence of chaotic behaviour in the time series data. Similar analyses were carried out for all the data segments corresponding to the 3-month periods of the years 2001 and 2007. The computed values of $K$ with $N=20000$ for all the cases are shown in Table 1. Also shown in this table are the values of the largest Lyapunov exponent (LE) for these periods, and are discussed below.

The existence of a positive Lyapunov exponent is an indicator that determines the chaotic nature of the dynamics. Vassiliadis et al. (1991) calculated the Lyapunov exponents for the $A L$ time series and obtained positive values in the range of $0.06-0.17 \mathrm{~min}^{-1}$, which clearly indicated the presence of chaotic behaviour in the $A L$ time series. In this analysis of the $A L$ time series using the $0-1$ test, we have obtained the values of the $K$ parameter all approximately close to 1 , indicating the presence of chaotic behaviour. Thus, the results of analysis using the $0-1$ test are in good agreement with the earlier results (Vassiliadis et al., 1991). For a more direct comparison, the Lyapunov exponents are computed for the data segments shown in Fig. 2 using the TISEAN package (Hegger et al., 1999). Here the time series data are represented as a trajectory in an embedded space and the Lyapunov exponents are calculated by searching for all neighbours within 

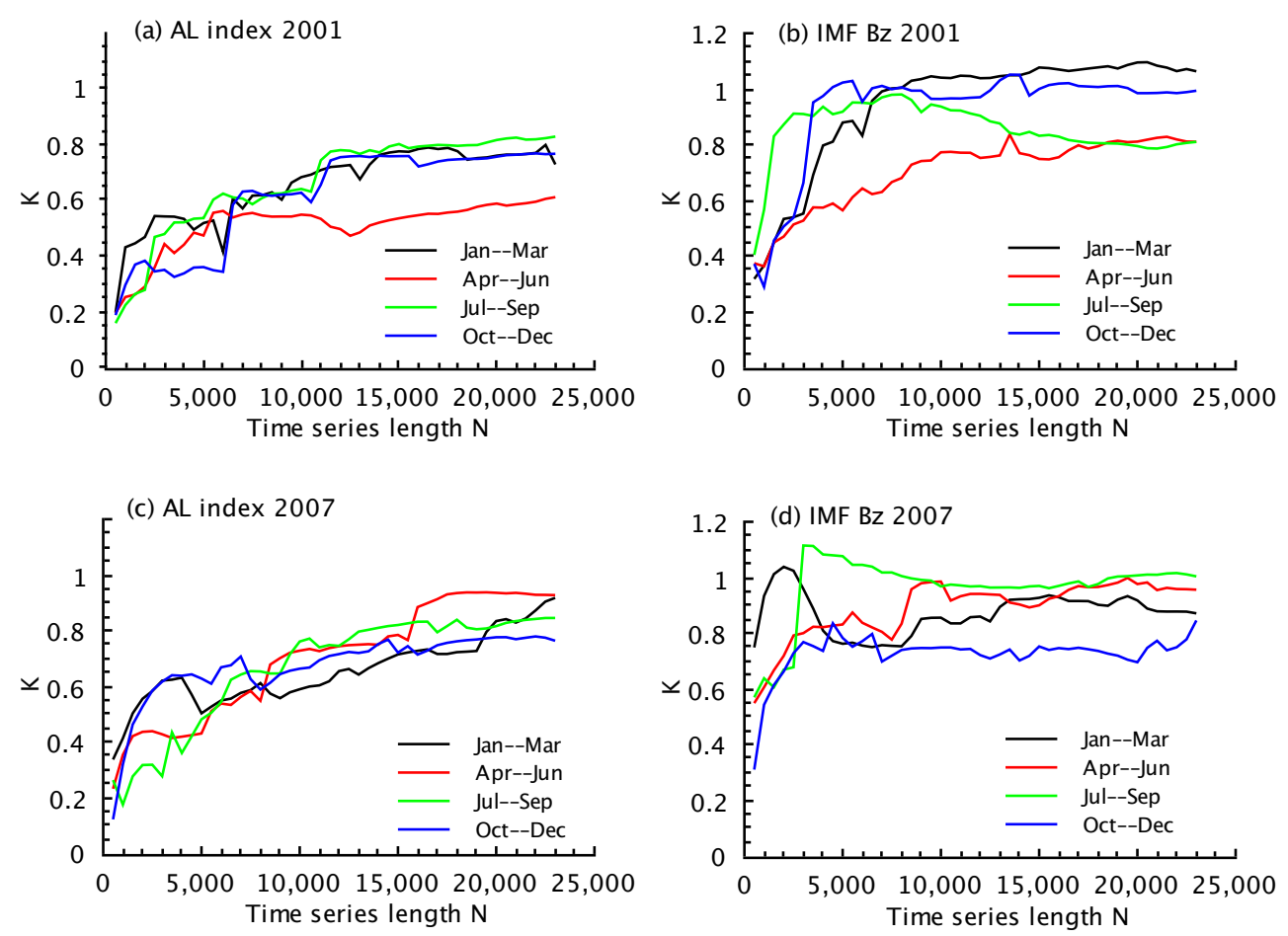

Fig. 2. The values of $K$ versus the time series length $N$ for different time windows of $A L$ index and IMF $B_{z}$ during 2001 and 2007.

(a) AL index 2001

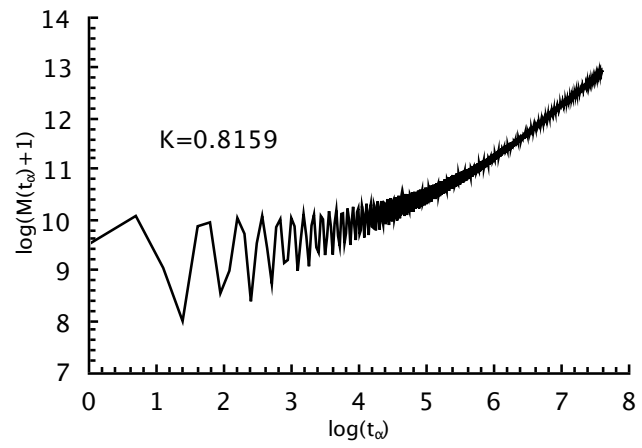

(c) AL index 2007

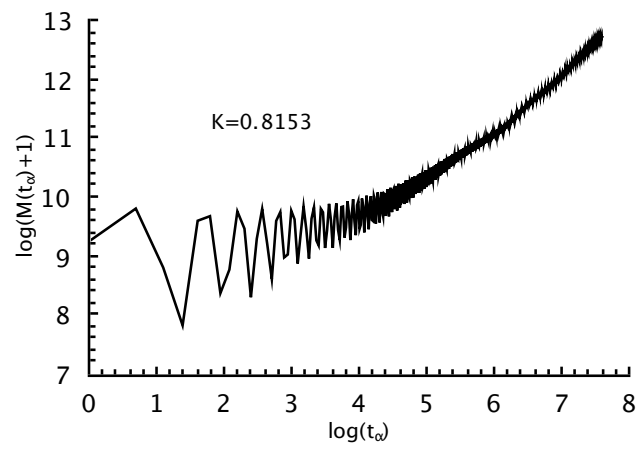

(b) IMF Bz 2001

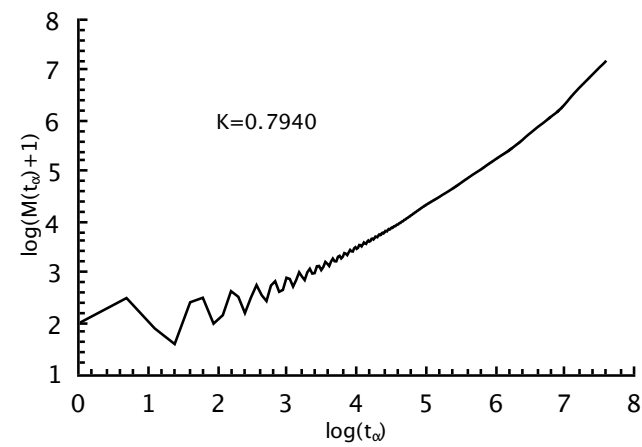

(d) IMF Bz 2007

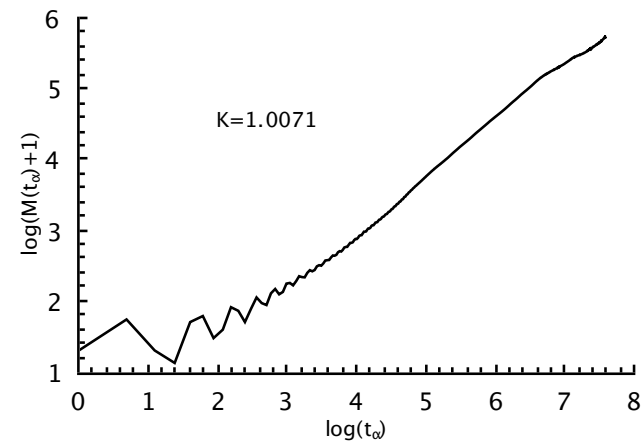

Fig. 3. The computation of $K$ values from the $\log \left(M\left(t_{\alpha}\right)+1\right)$ vs. $\log \left(t_{\alpha}\right)$ plots from the 5-min averaged $A L$ index and IMF $B_{z}$ during JulySeptember 2001 and 2007, using $N=20000$ data points. The slopes of the curves converge well, yielding the $K$ values. The cases for the other epochs have similar features. 
a neighbourhood of the reference trajectory as a function of time. We have embedded the time series data in four dimensions and used a delay of six for the purpose of computing the largest Lyapunov exponents. The Lyapunov exponents, shown in Table 1, agree well with the results of the $0-1$ test for the presence of chaos, and the values agree with the earlier values (Vassiliadis et al., 1991).

The $K$ values shown in Fig. 2 and Table 1 have similar values for the solar minimum (2001) and maximum (2007). This however does not mean that the levels of magnetospheric dynamics during these periods of very different driving by the solar wind are similar. The $0-1$ test provides a characterization of the inherent nature of the dynamics, viz. regular or chaotic, but does not quantify the differences in the details. This is especially true in the case of the solar windmagnetosphere system, which is a system far from equilibrium. This is also consistent with the results from the applications of the $0-1$ test to multiscale systems such as those with $1 / f^{\alpha}$ spectrum of fluctuations (Hu et al., 2005).

The results for the solar wind, shown in Fig. 2 and Table 1, show signatures of chaos, in terms of $K$ values being close to unity, for all the epochs. In fact the $K$ values are somewhat larger than those for the magnetosphere for the same periods, and they converge to these values at smaller data sizes (Fig. 2b and d). The $K$ values close to unity for the $A L$ data are consistent with the widely accepted view that the magnetosphere has coherent dynamical features arising from its internal dynamics, often referred to as the loading-unloading behaviour. At the same time the magnetosphere has features that are closer to turbulence, which are directly driven by the solar wind. The 0-1 test does not differentiate these features but is able to extract the dynamical features co-existing with the noisy behaviour. On the other hand the solar wind is turbulent and the $K$ values close to unity indicating deterministic dynamics seem contradictory at a first glance. However, turbulence in a wider sense is viewed as a high-dimensional dynamical system that manifests deterministic chaos (Bohr et al., 1998). Thus the data of a turbulent system are expected to yield features of dynamical chaos and the results for the solar wind magnetic field $B_{z}$ can be used in these terms. The 0-1 test thus detects the presence of deterministic dynamics but does not distinguish low dimensionality from the case of high-dimensional systems. Since most extended systems in nature are high dimensional, this limits the effectiveness of the $0-1$ test.

\section{Discussion}

The application of the $0-1$ test to the magnetosphere yields clear features of deterministic dynamics, with $K$ values close to unity. This is consistent with the earlier results on the low dimensionality of the magnetosphere (Sharma, 1995), which provided the basis for developing low-dimensional models for the prediction of the dynamics (Vassiliadis et al., 1995; Ukhorskiy et al., 2004; Chen and Sharma, 2006). However the magnetosphere is a far from equilibrium system and the observed features cannot be interpreted as a deterministic system alone. Such driven nonequilibrium systems exhibit global features that allow dynamical modelling and at the same time show multiscale features (Consolini and Chang, 2001; Ukhorskiy et al., 2004; Zelenyi and Milovanov, 2004). Also the magnetosphere exhibits long-range correlations (Sharma and Veeramani, 2011), and for such systems the $0-1$ test has limitations in providing the detailed features (Hu et al., 2005). Thus, while the results of the $0-1$ test by themselves may not be conclusive, it provides additional evidence of the deterministic aspects of the magnetosphere. In the studies of open systems, the framework of self-organized criticality has been used often and the magnetosphere has been viewed as such an avalanching system (Chapman et al., 1998; Consolini, 1997; Uritsky and Pudovkin, 1998; Uritsky et al., 2002). The consistency of this scenario with the results presented here is not clear as self-organized critical systems do not have positive Lyapunov exponents characterizing exponential divergence of nearby trajectories (Chen, 1990). However it is nontrivial to isolate such features from the data of systems far from equilibrium, such as the magnetosphere.

The database used in this study corresponds to periods of strong and weak geomagnetic activity, as represented by the years 2001 and 2007, corresponding to the last solar maximum and minimum, respectively. The magnetosphere is strongly driven by the intense solar activity during the solar maximum and exhibits strong coupling to the solar wind, resulting in better predictability (Chen and Sharma, 2006). However the $0-1$ test does not provide significant details of the differences between these two periods of widely different activity levels.

Acknowledgements. The authors gratefully acknowledge the CDAWeb at Space Physics Data Facility, http://cdaweb.gsfc.nasa.gov/istp_public/ for the OMNI data set used in the present study. The research at the University of Maryland was supported by NSF grants DMS 0417800 and AGS 1036473.

Edited by: G. Lapenta

Reviewed by: two anonymous referees

\section{References}

Abarbanel, H. D. I., Brown, R., Sidorowich, J. J., and Tsimring, L. S.: The analysis of observed chaotic data in physical systems, Rev. Mod. Phys., 65, 1331-1392, 1993.

Akasofu, S. I.: Energy coupling between the solar wind and the magnetosphere, Planet Space Sci., 28, 121-190, doi:10.1007/BF 00218810, 1981.

Barrow, J. D. and Levin J.: A test of a test for chaos, arXiv:nlin/0303070v1, available at http://arxiv.org/abs/ nlin0303070, 2003. 
Bohr, T., Jensen, M. H., Paladin, G., and Vulpiani, A.: Dynamical Systems Approach to Turbulence, Cambridge University Press, Cambridge, UK, 1998.

Chang, T.: Self organized criticality, multifractal spectra, sporadic licalized reconnections and intermittent turbulence in the magnetotail, Phys. Plasmas, 6, 4137-4145, 1999.

Chapman, S. C., Watkins, N. W., Dendy, R., Helander, R., and Rowlands, G.: A simple avalanche model as an analogue of magnetospheric activity, Geophys. Res. Lett., 25, 2397-2400, 1998.

Chen, J. and Sharma, A. S.: Modelling and prediction of the magnetospheric dynamics during intense geospace storms, J. Geophys. Res., 111, A04209, doi:10.1029/2005JA011359, 2006.

Chen, Z.-Y.: Noise-induced instability, Phys. Rev. A, 42,58375843, 1990.

Chowdhury, D. R., Iyengar, A. N. S., and Lahiri, S.: Gottwald Melborune (0-1) test for chaos in a plasma, Nonlin. Processes Geophys., 19, 53-56, doi:10.5194/npg-19-53-2012, 2012.

Consolini, C. and Chang, T. S.: Magnetic field topology and criticality in geotail dynamics: Relevance to substorm phenomena, Space Sci. Rev., 95, 309-321, 2001.

Consolini, G.: Sandpile cellular automata and the magnetospheric dynamics, in: Cosmic Physics in the year 2000, Proceedings of VIII GIFCO Conference, edited by: Aiello, S., Lucci, N., Sironi, G., Treves, A.. and Villante, U., 123 pp., SIF, Bologna, 1997.

Consolini, G., Marcucci, M. F., and Candidi, M.: Multifractal structure of auroral electrojet index data, Phys. Rev. Lett. 76, 40824085, 1996.

Falconer, I., Gottawald, G. A., Melbourne, I., and Wormnes, K.: Application of the 0-1 test for chaos to experimental data, SIAM J. Appl. Dyn. Syst., 6, 395-402, 2007.

Feldstein, Y. I., Popov, V. A., Cumnock, J. A., Prigancova, A., Blomberg, L. G., Kozyra, J. U., Tsurutani, B. T., Gromova, L. I., and Levitin, A. E.: Auroral electrojets and boundaries of plasma domains in the magnetosphere during magnetically disturbed intervals, Ann. Geophys., 24, 2243-2276, doi:10.5194/angeo-242243-2006, 2006.

Freeman, M. P., Watkins, N. W., and Riley, D. J.: Evidence for a solar wind origin of the power law burst lifetime distribution of AE indices, Geophys. Res. Lett., 27, 1087-1090, 2000.

Gottawald, G. A. and Melbourne, I.: A new test for chaos in deterministic systems, Proc. R. Soc. London A, 460, 603-611, doi:10.1098/rspa.2003.1183, 2004.

Gottawald, G. A. and Melbourne, I.: Testing for chaos in deterministic systems with noise, Physica D, 212, 100-110, 2005.

Hegger, R., Kantz, H., and Schrieber, T.: Practical implementation of nonlinear time series method: the TISEAN package, Chaos, 9, 413-430, 1999.

Hu, J., Tung, W.-W., Gao, J., and Cao, Y.: Reliability of the 0-1 test for chaos, Phys. Rev. E, 72, 056207, doi:10.1103/PhysRevE.72.056207, 2005.

Kantz, H. and Schrieber, T.: Nonlinear Time Series Analysis, Cambridge University Press, Cambridge, UK, 1997.

Kaplan, D. T. and Glass, L.: Direct test for determinism in a time series, Phys. Rev. Lett., 68, 427-430, 1992.

Kennel, M. B., Brown, R., and Abarbanel, H. D. I.: Determining embedding dimension for phase-space reconstruction using a geometrical construction, Phys. Rev. A, 45, 3403-3411, 1992.

Klimas, A., Vassiliadis, D., Baker, D., and Roberts, D.: The organized nonlinear dynamics of the magnetosphere, J. Geophys.
Res., 101, 13089-13113, doi:10.1029/96JA00563, 1996.

Klimas, A. J., Valdivia, J. A., Vassiliadis, D. V., Baker, D. N., Hesse, M., and Takalo, J.: Self organized criticality in the substorm phenomenon and its relation to localized reconnections in the magnetospheric plasma sheet, J. Geophys. Res., 105, 18765-18780, 2000.

Lorenz, E. N.: Predictability - a problem partly solved, in: Predictability of Weather and Climate, European Centre for Medium Range Weather Forecasting, edited by: Palmer, T., Shinfield park, Reading, UK, 1996.

Mayaud, P. N.: Derivation, Meaning and usage of geomagnetic Indices, Geophysical Monograph 22, American Geophysical Union, Washngton, DC, 1980.

Nicol, M., Melbourne, I., and Ashwin, P.: Euclidean extensions of dynamical systems, Nonlinearity, 14, 275-300, 2001.

Pavlos, G. P., Diamandidis, D., Adamopoulos, A., Rigas, A. G., Daglis, I. A., and Sarris, E. T.: Chaos and magnetospheric dynamics, Nonlin. Processes Geophys., 1, 124-135, doi:10.5194/npg-1-124-1994, 1994.

Pavlos, G. P., Athanasiu, M. A., Kugiumtzis, D., Hatzigeorgiu, N., Rigas, A. G., and Sarris, E. T.: Nonlinear analysis of magnetospheric data Part I. Geometric characteristics of the AE index time series and comparison with nonlinear surrogate data, Nonlin. Processes Geophys., 6, 51-65, doi:10.5194/npg-6-51-1999, 1999.

Price, C. P. and Prichard, D.: The nonlinear response of the magnetosphere, Geophys. Res. Lett., 20, 771-774, 1993.

Price, C. P., Prichard, D., and Bischoff, J. E.: Nonlinear input/output analysis of the auroral electrojet index, J. Geophys. Res., 99, 13227-13238, 1994.

Shan, L. H., Hansen, P., Goertz, C. K., and Smith, R. A.: Chaotic appearance of the AE index, Geophys. Res. Lett., 18, 147-150, 1991.

Sharma, A. S.: Assessing the Magnetosphere's Nonlinear Behavior: Its Dimension is Low, its Predictability High, (US National Rep. to the IUGG (1991-1994)), Rev. Geophys., 33, 645-650, 1995.

Sharma, A. S. and Veeramani, T.: Extreme events and long-range correlations in space weather, Nonlin. Processes Geophys., 18, 719-725, doi:10.5194/npg-18-719-2011, 2011.

Sharma, A. S., Vassiliadis, D., and Papadopoulos, K.: Reconstruction of low-dimensional magnetospheric dynamics by singular spectrum analysis, Geophys. Res. Lett., 20, 355-358, 1993.

Sharma, A. S., Baker, D. N., Grande, M., Kamide, Y., Lakhina, G. S., McPherron, R. M., Reeves, G. D., Rostoker, G., Vondrak, R., and Zelenyi, L.: The storm-substorm relationship: Current understanding and outlook, in: Disturbances in Geospace: The Storm-Substorm Relationship, Geophysical Monograph Series, Vol. 142, edited by: Sharma, A. S., Kamide, Y., and Lakhina, G. S., Amer. Geophys. Union, 1-14, 2003.

Sitnov, M. I., Sharma, A. S., Papadopoulos, K., Vassiliadis, D., Valdivia, J. A., Klimas, A. J., and Baker, D. N.: Phase transition-like behaviour of the magnetosphere during substorms, J. Geophys. Res., 105, 12955-12974, 2000.

Sitnov, M. I., Sharma, A. S., Papadopoulos, K., Vassiliadis, D., Valdivia, J. A., Klimas, A. J., and Baker, D. N.: Modeling substorm dynamics of the magnetosphere: from self-organization and selforganized criticality to non-equillibrium phase transitions, Phys. Rev. E, 65, 16116, doi:10.1103/PhysRevE.65.016116, 2001. 
Sun, K., Xuan, L., and Zhu, C.: The 0-1 test algorithm for chaos and its applications, Chin. Phys. B19, 110510, doi:10.1088/16741056/19/11/110510, 2010.

Ukhorskiy, A. Y., Sitnov, M. I., Sharma, A. S., and Papadopoulos, K.: Global and multiscale dynamics of the magnetosphere: From modeling to forecasting, Geophys. Res. Lett., 31, L0880208805, 2004.

Uritsky, V. M. and Pudovkin, M. I.: Low frequency $1 / f$-like fluctuations of the AE-index as a possible manifestation of selforganized criticality in the magnetosphere, Ann. Geophys., 16, 1580-1588, doi:10.1007/s00585-998-1580-x, 1998.

Uritsky, V. M., Klimas, A. J., Vassiliadis, D., Chua, D., and Parks, G. D.: Scale free statistics of spatio-temporal auroral emissions as depicted by POLAR UVI images: The dynamic magnetosphere as an avalanching system, J. Geophys. Res., 107, 1426, doi:10.1029/2001JA000281, 2002.

Valdivia, J. A., Sharma, A. S., and Papadopoulos, K.: Prediction of Magnetic Storms Using Nonlinear Models, Geophys. Res. Lett., 23, 2899-2892, 1996.
Vassiliadis, D. V., Sharma, A. S., Eastman, T. E., and Papadopoulos, $\mathrm{K}$.: Low-dimensional chaos in magnetospheric activity from $\mathrm{AE}$ time series, Geophys. Res. Lett. 17, 1841-1844, 1990.

Vassiliadis, D. V., Sharma, A. S., and Papadopoulos, K.: Lyapunov exponent of magnetospheric activity from AL time series, Geophys. Res. Lett., 18, 1643-1646, 1991.

Vassiliadis, D. V., Klimas, A. J., Baker, D. N., and Roberts, D. A.: A description of solar wind-magnetosphere coupling based on nonlinear filters, J. Geophys. Res., 100, 3495-3512, 1995.

Vasyliunas, V. M.: Theoretical models of magnetic field line merging, Rev. Geophys. Space Phys., 13, 303-336, 1975.

Wayland, R., Bromley, D., Pickett, D., and Passamante, A.: Recognizing determinism in a time series, Phys. Rev. Lett. 70, 580-582, 1993.

Zelenyi, L. M. and Milovanov, A. V.: Fractal topology and strange kinetics: from percolation theory to problems in cosmic electrodynamics, Phys. Usp., 47, 749-788, 2004. 